\title{
Apport de l'approche par milieux innovateurs pour comprendre et penser le développement territorial : cas de l'innovation patrimoniale
}

\author{
Hassan EDMAN \\ Laboratoire d'études et de recherches appliquées en sciences économiques (LERASE) \\ Faculté des sciences juridiques, économiques et sociales \\ Université Ibn Zohr, Agadir, Maroc
}

\begin{abstract}
Résumé : Cet article se propose d'étudier l'apport de l'approche par milieux innovateurs aux politiques de développement territorial. Sur les trente dernières années, l'approche a permis un nouveau regard et de profonds renouvellements d'hypothèses et de problématiques de l'analyse territoriale. Elle permet de mieux lier le comportement innovateur des acteurs locaux à la dynamique économique, originellement, dans le sens que c'est le territoire qui secrète l'innovation et l'entrepreneuriat innovant. Nous proposons une réflexion croisée entre la notion de "ressource patrimoniale" et le référentiel théorique de base, les milieux innovateurs. Il s'agit notamment de présenter le caractère innovateur des milieux producteurs à base de ressources patrimoniales, les configurations possibles de l'inscription territoriale des ressources ainsi qu'une typologie de dynamiques qui se créent et qui font émerger de nouvelles combinaisons d'éléments, potentiellement pertinentes ou handicapantes pour un développement territorial durable.
\end{abstract}

Mots-clés : dynamique économique, système de production, milieu innovateur, ressource patrimoniale.

\section{Introduction}

Dans un contexte de globalisation, tous les systèmes territoriaux de production quelle que soit leur composition, doivent innover et chercher de nouvelles ressources pour maintenir leurs avantages compétitifs à long terme. C'est dans l'obligation de survivre que les territoires doivent innover, surtout que plus les économies s'ouvrent, plus la compétitivité territoriale devient féroce et intense.

L'innovation permet de rattraper le retard de développement et de repousser l'état stationnaire de l'économie par des "grappes d'innovation". Elle permet pareillement d'avoir des rendements croissants de la croissance économique. L'innovation est un choix de politiques publiques, autour duquel se tracent des trajectoires de développement territorial.

A cet égard, l'approche par milieux innovateurs est la plus adéquate pour analyser ce lien causal réciproque entre l'innovation et les transformations économiques du territoire. Et ce, pour son "réalisme scientifique" vis-à-vis la réalité du territoire, qui revient au nombre hautement important des études empiriques menées par le groupe de recherche européen sur les milieux innovateurs (GREMI), sur la base desquelles l'approche s'est construite. C'est inévitable vu que, "Le programme de recherche mené par le GREMI jusqu'à aujourd'hui se caractérise précisément par une interaction étroite et systématique 
entre le travail de terrain et la théorisation. Chaque enquête, en amenant des exemples et des contreexemples, a permis de faire évoluer le concept de milieu innovateur." ${ }^{1}$

Ses fondements aussi que son apport à l'explication des dynamiques économiques, vont être exposés dans un premier temps. Ensuite, nous développons la notion de l'innovation patrimoniale, portant des éclaircissements quant aux deux notions "ressources naturelles" et "ressources culturelles" et nous finissons par l'analyse des dynamiques économiques favorisées par l'innovation patrimoniale et des différentes configurations et formes d'inscription territoriale des ressources patrimoniales.

\section{Nouveau fondement, nouvelles hypothèses}

\subsection{L'approche par "Milieu", les origines et les fondements}

L'approche par "Milieu" tire une partie de ses origines de celles de la science régionale. Principalement, les faiblesses des analyses économiques quant à la prise en compte de l'espace et l'irréalisme des paradigmes de l'économie classique. D'une part, l'analyse économique classique est faussée par le paradigme de la rationalité parfaite, d'autre part, même si la dimension spatiale est intégrée, bien aussi dans l'approche par "territoire", l'intégration prend souvent forme de coûts de distance, en deuxième temps, après que l'analyse économique se fait, en premier temps, hors son contexte spatio-temporel.

Souvent, l'analyse économique cherche des réponses aux problématiques du développement territorial en dehors du facteur clé, celui de l'organisation économique et l'inter-connectivité des acteurs sur le territoire. De la sorte, elle dépossède ces derniers de leur rôle dans la mise en œuvre des structures porteuses de développement.

La science régionale s'est développée au moyen des outils d'analyse de la micro et de la macro-économie, qui sont issues du paradigme de la "rationalité parfaite". D'où la nécessité d'une révision et une reconstruction de la science régionale en dehors du paradigme fondateur de la science économique, celui de "la rationalité parfaite".

Pour ce qui nous importe par rapport à notre sujet, l'innovation et le développement sont fréquemment analysés isolément et non pas comme des processus collectifs qui s'alimentent mutuellement. Les modèles d'innovation et de développement utilisés ne considèrent pas les processus organisationnels et de la création technologique. C'est exactement ce que propose l'approche par "milieu" dont l'apport distinctif réside dans sa capacité de tenir compte des aspects spatiaux et organisationnels des transformations économiques. Encore plus, c'est dans l'optique inverse que l'apport est essentiel et innovant. C'est-à-dire, en quoi, le territoire/milieu contribue à la construction des dynamiques économiques?

Le "Milieu" oriente l'analyse vers les formes de gouvernance collectives qui sont à la base de la construction socio-économique et ouvre l'analyse à la coopération, et donc une nouvelle analyse de la dynamique économique. Plus précisément, le "Milieu" considère la "dynamique organisationnelle" qui fait que le développement socio-économique n'est plus résultat d'une rationalité issue de mécanismes préexistants, mais qu'il s'auto-évolue et s'auto-organise en construisant sa propre rationalité.

En effet, la construction socio-économique du territoire est une démarche complexe à travers laquelle une société forge ses capacités et ses propres mécanismes d'autogenèse organisationnelle. C'est ainsi que la

\footnotetext{
${ }^{1}$ Olivier Crevoisier, « L'approche par les milieux innovateurs : état des lieux et perspectives », 154.
} 
communauté locale constitue son propre "Milieu" à partir de la totalité territoire-culture-conscience collective.

Le territoire "milieu" est un espace constitué d'un ensemble de relations entre les hommes et entre les hommes et leur environnement matériel. Sur le "milieu", les éléments constitutifs ne sont pas forcément tous inter-reliés, il y a ce qui est relié au système et ce qui est délié et isolé. Aussi, il se spécifie et se façonne par une culture qui influence les finalités, les modalités et la nature de l'organisation économique entre les acteurs moteurs. Assurément, l'approche par les milieux remet en avant la dimension culturelle de la création socio-économique.

L'approche par milieu parait être le cadre théorique idoine permettant d'analyser les conditions par lesquelles un contexte socio-économique territorial se cristallise en une forme organisationnelle du milieu, qui fait émerger une autonomie et des changements endogènes. Elle permet ainsi d'expliquer et de justifier comment un système local devient un "milieu innovateur".

Au fils du temps, un territoire milieu devient innovateur par "la mobilisation des ressources constituées par le passé, adaptées aux nouvelles techniques et nouveaux marchés, et incorporées dans de nouveaux produits" 2 . Et ce, par des ruptures/filiations ${ }^{3}$ qui permettent une différentiation progressive des composants du milieu, par rapport à l'environnement.

Les milieux innovateurs se caractérisent par leur savoir-faire de coopération créatrice de performance économique et par la culture industrielle collective qui valorise les systèmes de production locaux. A noter un deuxième trait distinctif, aux milieux innovateurs "les spécialisations contribuent au développement, en introduisant dans les structures productives un supplément de rationalité technologique et organisationnelle"4. Outre la spécialisation, le perfectionnement des savoirs acquis est un deuxième facteur stimulant de la dynamique économique.

\subsection{Le renouveau de la problématique et l'approfondissement des hypothèses pour une compréhension meilleure des dynamiques spatiales}

Au cœur de l'approche par les milieux innovateurs, l'interdépendance consubstantielle entre la théorie et la réalité était le trait fort de tous les travaux menés par GREMI (Groupe de recherche européen sur les milieux innovateurs) depuis 1986 avec comme hypothèse de départ : Il y’a quelques choses localisées au niveau régional, permettaient de comprendre pourquoi certaines régions étaient plus dynamiques qu'aux autres.

L'approche s'articule autour de trois axes d'investigation qui permettent de mieux appréhender le milieu innovateur : la dynamique technologique, la transformation des territoires, et le changement organisationnel. "Milieux innovateurs" est un concept intégrateur d'analyse et de compréhension des transformations économiques, de trois positions paradigmatiques :

- Paradigme technologique : permet de comprendre le rôle des techniques de l'innovation (concurrence par la différenciation) dans les transformations. Etant donné que le développement

\footnotetext{
${ }^{2}$ Olivier Crevoisier, "L'approche par les milieux innovateurs : état des lieux et perspectives », 158.

${ }^{3}$ Le processus qui met en jeu d'interactions d'une part, le milieu, qui offre les ressources (savoir-faire, capital relationnel, etc.), et les réseaux d'innovation d'autre part, qui en sont la mobilisation et l'actualisation à travers un processus d'innovation

${ }^{4}$ Jean-Claude Perrin, "Pour une révision de la science régionale. L'approche par les milieux », 180.
} 
de nouvelles techniques, pratiques et produits est une manière de préserver la compétitivité des espaces.

Cependant, l'innovation ne peut plus être réduite à la $R \& D$ ou aux dépôts de brevets. Techniquement, l'innovation peut se comprendre comme le résultat de l'articulation des ressources de l'entreprise ou de son environnement. La mise de nouvelles techniques et de nouveaux produits sur le marché conduit à l'émergence de nouveaux savoir-faire, enclenchant des processus d'apprentissage.

- Paradigme organisationnel : ce sont les mécanismes qui permettent ou empêchent la coordination entre acteurs et articulent en même temps les attributions fonctionnels et territoriaux. Analyser ce paradigme c'est décrire les règles de concurrence/coopération locales.

En effet, les réseaux locaux d'acteurs contribuent également au maintien ou à la reproduction des frontières entre le milieu et l'extérieur. La compétitivité du milieu résulte plutôt de sa capacité particulière de coordination et de gestion des tensions et de son pouvoir organisationnel d'adaptation.

Au cours du temps, le fonctionnement des milieux génère des interdépendances non marchandes, "un collectif" capital relationnel. A signaler, le cadre organisationnel appelé "gouvernance" (Stoker 1998) renvoie aux mécanismes de conduite d'une structure ou d'un ordre qui ne sont pas imposés de l'extérieur, mais résulte de l'interaction d'un certain nombre de groupes qui s'influencent mutuellement.

- Le paradigme territorial : le territoire génère les ressources (savoir-faire, compétences...) et les acteurs (entreprises, innovateurs, institutions de support...) nécessaires à l'innovation. Aussi, les savoir-faire sont des ressources spécifiques au territoire, reproduites par l'activité économique et par les différentes institutions de formation, de recherche, de soutien et autres. Les capacités locales sont des construits locaux du territoire.

L'approche pose comme ontologiquement identiques, les trois paradigmes. Il n'y a pas d'hiérarchie, chacune des trois dimensions permet de saisir le milieu différemment, mais en étroite interaction avec les deux autres. Dans le même cadre, l'approche par milieux innovateurs appréhende la réalité complexe du milieu et de son fonctionnement, par prise en compte des dualités, proximité/distance, concurrence/coopération et innovation/tradition, dans l'analyse. 


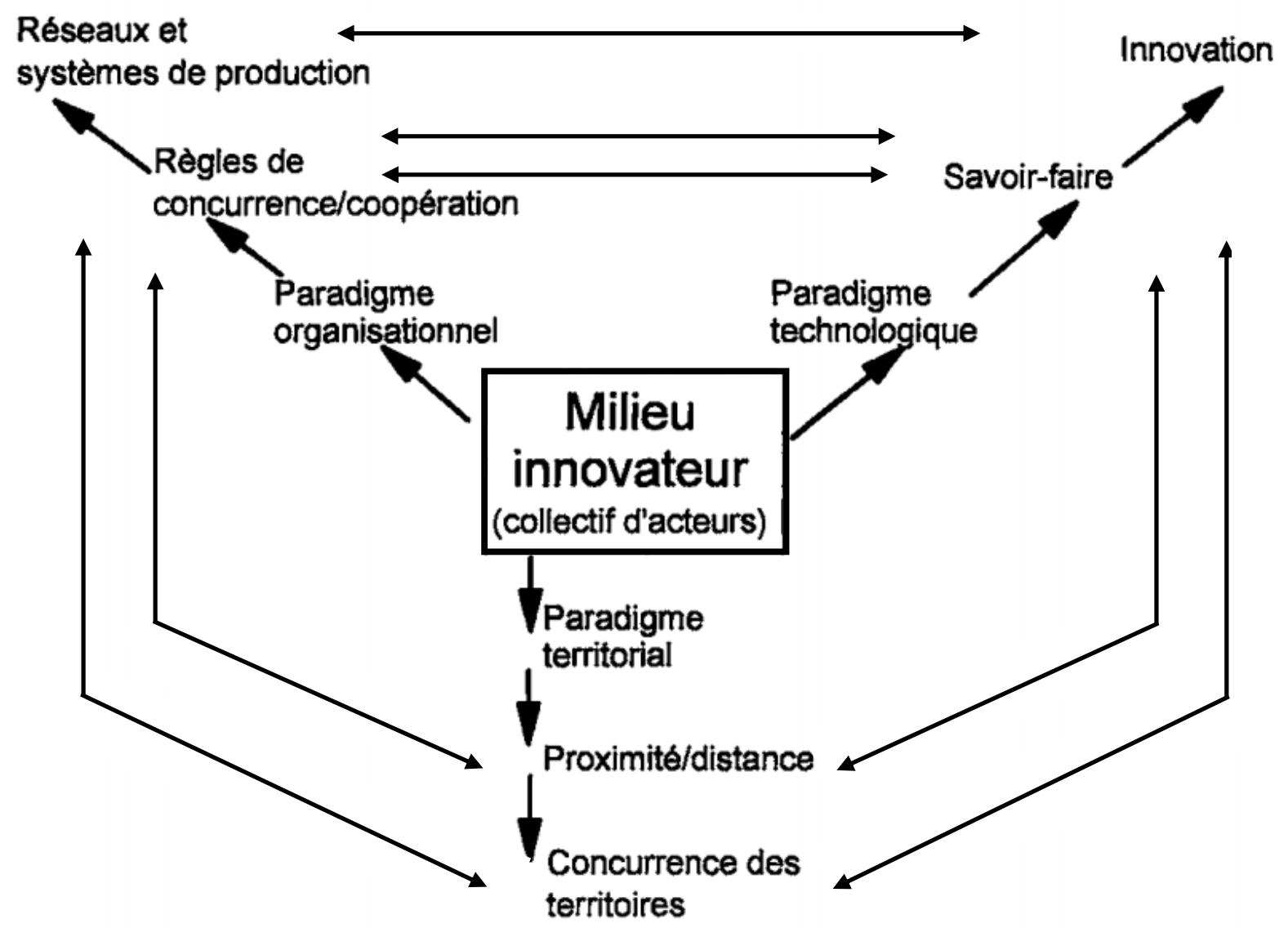

Figure 1: Les paradigmes des milieux innovateurs et le développement économique territorialisé Source : Olivier Crevoisier, 2001

Fort à signaler, l'originalité de cette approche est qu'elle considère ces trois paradigmes indispensables, simultanés et égaux. Comme d'ailleurs ce n'est pas le cas en économie industrielle où c'est la dynamique industrielle qui prime et qui produit le territoire. Pareillement en géographie, c'est l'espace qui secrète les dynamiques urbanistes et économiques.

La problématique autour de laquelle oscillent tous les travaux sur les milieux innovateurs, cherche des explications quant aux inégalités dans les trajectoires de développement régionale. Ainsi, l'approche par milieux innovateurs permet de comprendre les dynamiques spatiales notamment des régions décrites souvent "innovatrices" ou "à succès", et d'expliquer l'échec des régions "bloquées". C'est donc un nouveau regard sur ces dynamiques qui les considère, les qualifie et les systématise dans leur spatialité. Un territoire est normativement qualifié milieu innovateur par l'identification d'un modèle de développement économique territorialisé, caractérisé par :

- Une concurrence par l'innovation et non pas les coûts de production.Une organisation par systèmes productifs et non sur des mécanismes de marché ou hiérarchiques.

- Une concurrence axée entre territoires et non pas entre entreprises.

Toutefois, GREMI a travaillé aussi sur les convergences et les similitudes existantes entre la ville et le milieu innovateur. En effet, les dynamiques des grandes villes, les " métropoles" au sens de Veltz (1996) 
ou "Global cities" de Sassen (1991) et Crevoisier, Gamagni (2000) divisent le territoire de la ville en des systèmes de production organisés comme des milieux. A titre d'exemple, les milieux et les villes ont en commun :

- La proximité et sa mise en profit.

- Les opportunités de créativité.

- La capacité d'agir et de penser en réseau et d'articuler les deux échelles, locale et globale.

- La présence de l'élément relationnel et synergétique (CAMAGNI, 2000).

- La concurrence par différenciation.

- L'organisation par systèmes productifs et non sur des mécanismes de marché ou hiérarchiques.

- La compétitivité territoriale dans l'intégralité du territoire et non seulement par ses entreprises.

\section{Milieux innovateurs et dynamique régionale}

\subsection{Milieux innovateurs, une dynamique territoriale qui secrète de l'innovation}

Aux années 80, Philipp Aydalot identifie de nouveaux dynamismes et des transformations structurelles fortement liés au progrès technique. A l'opposé de la conception dominante qui considère que l'innovation émerge uniquement au sein des grandes entreprises puis se diffuse à l'intérieur de l'économie, Aydalot avance l'hypothèse selon laquelle les milieux territoriaux et non pas les entreprises qui innovent, et propose ainsi le concept de "milieux innovateurs".

Tous les travaux du GREMI ont été axés sur l'identification des milieux innovateurs à travers leur capacité à susciter l'innovation dans les systèmes territoriaux de production. Par "milieu innovateur", GREMI désigne " l'ensemble territorialisé dans lequel des interactions entre agents économiques se développent par l'apprentissage, qu'ils font de transactions multilatérales génératrices d'externalités spécifiques à l'innovation et par la convergence des apprentissages vers des formes de plus en plus performantes de gestion en commun des ressources"5. L'innovation est donc considérée par le groupe de recherche GREMI, comme étant un processus où maints éléments interagissent et s'alimentent réciproquement sur le territoire, et favorisent la dynamique du système productif.

Encore dans le même cadre, la capacité d'innover d'un territoire ne dépend pas d'une dotation en facteurs, mais de son passé, du comportement des acteurs locaux, des capacités de prise de l'initiative, des processus décisionnels et des tensions entre acteurs locaux. GREMI focalise son attention sur les modalités organisationnelles et territoriales de l'émergence de l'innovation.

\subsection{Quel entrepreneuriat pour quelle dynamique ?}

Le rôle de l'entrepreneuriat comme créatrice de la croissance économique était théorisé, accent mise, sur l'importance de la contribution des multinationales et des grandes entreprises dans la dynamisation de l'économie et notamment la stimulation de l'innovation. Cet angle d'analyse développé par Schumpeter a donné explication au fonctionnement des économies surtout industrialisées.

\footnotetext{
${ }^{5}$ Denis MAILLAT, Michel Quévit, et Lanfranco Senn, « Réseaux d'innovation et milieux innovateurs : un pari pour le développement régional », 9.
} 
Cependant, depuis les années 1970, après les trente glorieuses, le nombre grandissant des PME ainsi que leur capacité de créativité et d'innovation, a réorienté le débat sur le rôle de nouvelles formes de production suivant deux logiques, fonctionnelle et territoriale. Dans une optique territoriale, "le dynamisme territorial ne dépend pas de l'action d'une seule entreprise, mais d'un comportement d'ensemble, fonction d'un réseau d'interdépendances qui se manifestent au niveau territorial"6.

L'analyse par milieux innovateurs permet de comprendre les dynamiques d'innovation des entreprises. En effet, "Les enquêtes du GREMI ont montré d'ailleurs que ces noyaux de relations se combinent entre les diverses phases du processus d'innovation (conception, production, commercialisation) et les différentes fonctions de l'entreprise (achat de marchandises ou d'équipement, vente des produits, gestion, acquisition de connaissances, etc.). L'entreprise n'est pas seulement innovatrice par sa capacité de créer une nouvelle technologie ou un nouveau produit, mais aussi par sa capacité de gérer la complexification croissante de ses échanges externes au niveau local et international"7.

Sous le même angle d'approche, les deux actes, entreprendre et innover dépendent de conditions et des ressources offertes par le milieu. Aydalot constate en 1986 que l'entreprise innovatrice ne préexiste pas sur le territoire mais sécrétée par celui-ci. Également, le comportement innovateur dépend de variables intrinsèques au territoire.

L'approche par milieux innovateurs permet de comprendre le rôle joué par la composante territoriale dans la création des entreprises et l'innovation d'une part, et l'encastrement de l'entrepreneuriat dans son environnement territorial.

L'attractivité des milieux innovateurs relève de son mode d'organisation, notamment les relations de proximité qu'ils assurent entre les différents acteurs, pouvoirs publics et institutions privées financières et de recherche. Ajoutant le couplage et l'articulation entre le local et le supra-local, offrant plus d'opportunités d'échanges, de financement et de commercialisation aux entreprises installées sur le milieu.

\footnotetext{
${ }^{6}$ Denis Maillat et Jean-Claude Perrin, « Entreprises innovatrices et développement territorial », 1992, 4.

${ }^{7}$ Michel Quévit, « Milieux innovateurs et couplage local- international dans les stratégies d'entreprise: un cadre pour l'analyse », 223.
} 


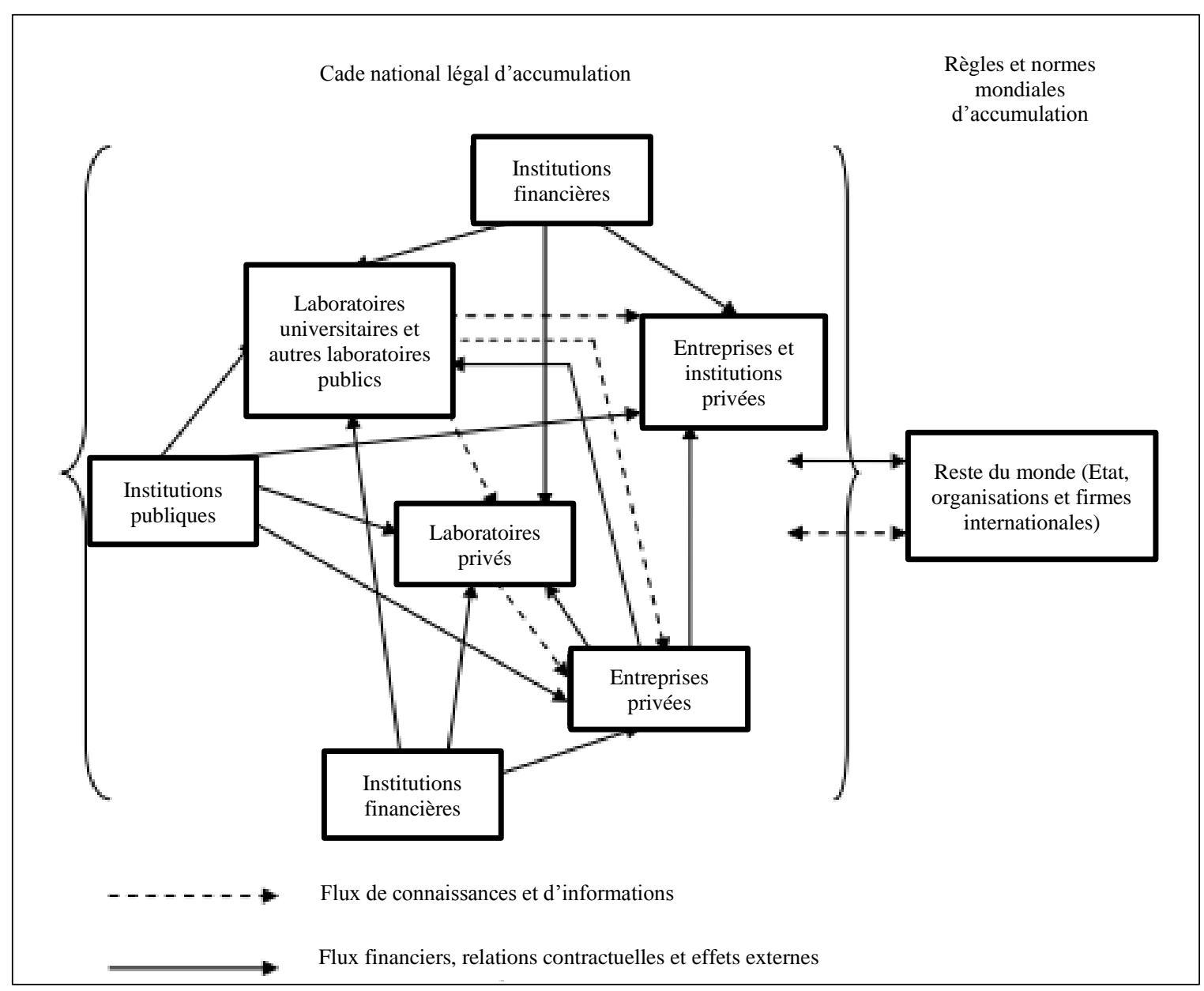

Figure 2 : Milieux innovateurs et entrepreneuriat

Source : Dimitri Uzunidis 2010

L'entreprise innovante est fortement ancrée à son milieu, son profil dépend des spécificités des systèmes productifs, innovateurs et territoriaux auxquels elle appartient. C'est une des singularités du milieu innovateur, en tant que dynamiseur de l'entrepreneuriat et à travers, de l'innovation.

Le milieu façonne et donne son empreinte à un entrepreneuriat spécifique. Le milieu innovateur se caractérise par sa capacité d'identifier, développer, reproduire et enrichir de manière autonome des savoir-faire favorisant une certaine spécialisation des systèmes producteurs.

Conséquemment, la création des entreprises et aussi le sens de l'innovation "collective" sont rarement l'émanation d'un acteur unique et isolé, ils sont des processus sociaux collectifs de haute complexité. Les relations de proximité générées par le fonctionnement du milieu innovateur font émerger et développent un entrepreneuriat innovant et innovateur. 


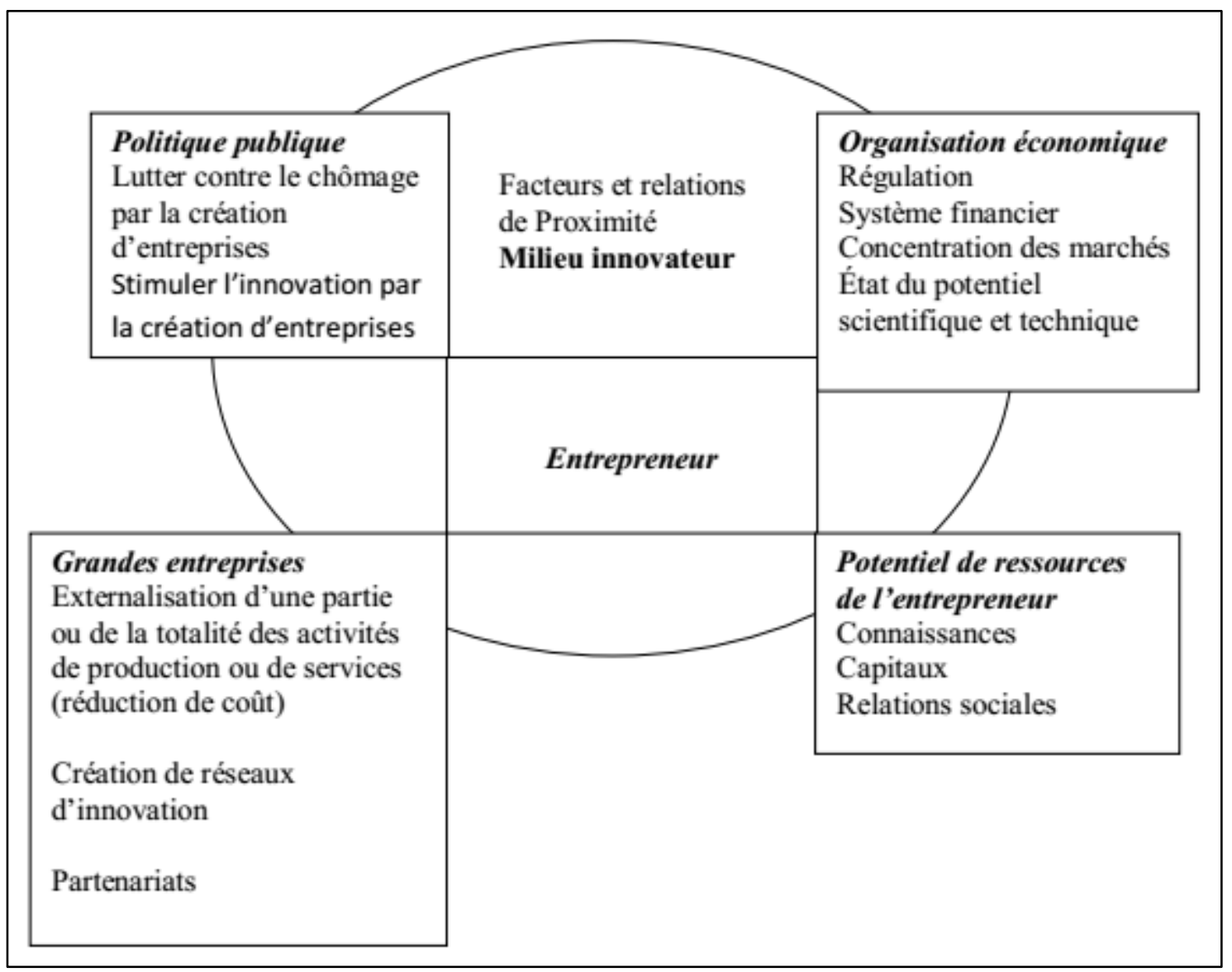

Figure 3 : L'entrepreneur au centre du milieu innovateur

La capacité à entreprendre résulte de la variété et de la richesse du réseau mobilisé par le milieu. Également, la composition du réseau, le cadre institutionnel du milieu, conditionnent la formation des capacités entrepreneuriales. La richesse qu'offre le milieu innovateur s'incarne dans des ressources de différentes formes.

Les politiques publiques décidées et menées par l'ensemble des institutions du milieu, font richesse et créent des opportunités à l'entrepreneur. L'affirmation est claire quant à la lutte contre le chômage, l'encouragement de l'initiative privée ou justement la stimulation de l'entrepreneuriat et de l'innovation.

De même pour l'organisation économique du milieu, dans le sens où il offre des débouchés, des institutions de financement, un réseau d'innovation et des grandes entreprises installées, représentant des partenaires ou des demandeurs éventuels de services dans le cadre de l'externalisation d'une partie de leurs fonctions.

Finalement, le milieu innovateur est une combinaison sur un espace donné d'institutions, d'opportunités et de ressources favorables à une dynamique entrepreneuriale qui contribue en retour au développement des capacités du milieu, à sécréter de nouvelles entreprises. En effet, le milieu innovateur relie trois forces entrepreneuriales, l'attractivité par l'effet d'agglomération qu'il produit, la diffusion et l'externalité par l'effet de dispersion et d'entrainement de ses agglomérations à "succès". Le milieu innovateur est plus protecteur du fait qu'il présente plus de garanties par les relations de proximité qu'il assure et donc moins de risques pour tout acte d'entrepreneuriat. 


\section{De l'innovation technologique à l'innovation patrimoniale par ressources naturelles et culturelles}

\subsection{Innovation patrimoniale et ressources naturelles et culturelles, précisions conceptuelles}

L'approche par milieux innovateurs s'est intéressée, dans le sixième projet de GREMI, à la problématique de la valorisation des ressources naturelles et culturelles. Quelle articulation entre les ressources patrimoniales et le développement des milieux producteurs locaux ? En quoi l'innovation patrimoniale diffère de l'innovation classique, issue de milieux industriels ou technologiques ?

Assurément, les enquêtes de GREMI VI ont eu pour objet de confronter le concept de milieux innovateurs à des systèmes de production ayant pour finalité l'exploitation d'un patrimoine. Il s'est fait en trois étapes :

- Identifier les ressources à partir desquelles de nouvelles dynamiques territoriales s'élaborent.

- Apprécier le rôle du milieu (existe ou non), les acteurs et leurs modes de gestion innovante de la ressource.

- Déterminer dans quelle mesure la ressource était gérée de manière à garantir sa durabilité.

\subsubsection{Qu'est ce qui fait ressource?}

Les ressources, comme étaient traitées depuis toujours par la pensée économique, sont à l'origine de la richesse. Naturelles et marchandes tirées de la terre pour les physiocrates ou issues des métaux précieux pour les mercantilistes. Ensuite, la ressource commence à être analysée en tant qu'input aux systèmes productifs. Facteur de production, comme d'autres, la ressource est saisie pour sa stricte capacité à satisfaire un besoin au sein du système de production, et donc pour son apport économique et pour la valeur qu'elle crée.

Pour GREMI, une distinction doit être faite entre la ressource/l'objet et la manière dont elle est inscrite dans un système productif, un territoire ou un milieu. C'est le lien et l'articulation entre la ressource et le système de production qui fait valeur et qui est l'objet de l'innovation du milieu.

En réalité, "les ressources ne sont plus considérées seulement pour elles-mêmes et pour leur capacité à s'intégrer dans une combinatoire donnée de facteurs mais, elles deviennent l'objet d'un processus de reproduction et de redéploiement, nous dirons de "requalification", par lequel la "spécification" des ressources prend forme et se renouvelle au fil du temps"8.

L'objet des ressources naturelles et culturelles, dans l'analyse territoriale, est naturellement associé au caractère de "patrimonialité" qui fait des ressources, des valeurs intrinsèques à sauvegarder. Un bien commun, le patrimoine naturel est composé de l'ensemble des ressources naturelles (de la nature vivante, ressources fossiles ou minérales, ressources halieutiques, ressources forestières, plantes, paysages...) présentes dans la nature, déjà identifiées et exploitées pour satisfaire les besoins du territoire, ou latentes.

De même, la ressource culturelle et aussi patrimoniale, évoque "l'ensemble des biens, matériels ou immatériels, ayant une importance artistique et/ou historique certaine, et qui appartiennent soit à une

\footnotetext{
${ }^{8}$ Véronique PEYRACHE-GADEAU et Bernard PECQUEUR, « Les ressources patrimoniales : une modalité de valorisation par les milieux innovateurs de ressources spécifiques latentes ou existantes », 73.
} 
entité privée (personne, entreprise, association, etc.) ${ }^{9}$, soit à une entité publique (commune, département, région, pays, etc.)". Parmi plusieurs catégorisations du patrimoine culturel nous retenons celle de l'UNESCO où la ressource culturelle prend deux formes, matérielle et immatérielle :

- Ressources culturelles matérielles : mobilières, exemples de la monnaie, des sculptures ou autres biens mobiles. Ou immobilières, exemples des monuments historiques

- Ressources culturelles immatérielles : des savoirs, des pratiques, des procédés traditionnels, des danses folkloriques, traductions populaires, des créations collectives...

\subsubsection{De l'innovation technologique à l'innovation patrimoniale}

L'objectif des enquêtes GREMI VI ${ }^{10}$ est d'analyser de nouveaux types de milieux organisés autour des ressources naturelles et culturelles passant ainsi du paradigme "innovation technologique" au paradigme "innovation patrimoniale". La mise en évidence de la rupture/filiation des réseaux d'innovation est aussi au cœur des projets de GREMI, notamment par l'analyse des ressources et des différences entre les deux types de développement : par progrès technique et par ressources. A noter que la question des ressources naturelle et culturelle se place dans une perspective évolutionniste.

Alors que l'innovation technique porte l'idée de progrès et de dynamisme et se calcule par la rapidité de l'obsolescence. Au contraire, les ressources naturelles et culturelles ont un caractère de durabilité et de quasi-immutabilité complètement antinomique de l'innovation.

Contrairement à l'innovation technologique qui se situe dans un univers marchand, la raison d'être d'un patrimoine, c'est d'être conservé dans le long terme, ce qui pose le problème des valeurs non marchandes et qui nécessite une démarche nouvelle pour la science économique qui ne connait que des valeurs monétaires. Le souci donc est de trouver l'équilibre délicat entre l'exploitation marchande de la ressource et son maintien dans la durabilité.

La territorialisation de l'exploitation des ressources permettra probablement cet équilibre, notamment par une exploitation à l'aune de milieux innovateurs qui combinent le développement durable en même temps que la préservation des capacités d'innovation.

C'est le rôle et l'apport original du milieu innovateur, le fait de réaliser cet équilibre délicat et non évident. Assurer la pérennité de l'exploitation des ressources naturelles et culturelles sans rater les opportunités de les mettre dans une machine productive qui risque de les épuiser. Un vrai dilemme à soulever!

Ces dernières considérations, nous laissent affirmer que l'innovation patrimoniale réside dans la capacité du milieu innovateur à mobiliser les ressources patrimoniales, pour en créer de la valeur et en dégager des dynamiques socio-économiques, en dehors de la logique purement marchande, souvent et irrationnellement consommatrice des ressources.

\subsection{L'innovation patrimoniale, les différentes configurations}

La mise en valeur d'un patrimoine culturel ou naturel dépend de la capacité d'un milieu à découvrir et à exploiter des ressources latentes ou délaissées. Innover en matière de ressources patrimoniales implique des transformations sociales et organisationnelles du territoire.

\footnotetext{
${ }^{9}$ https://fr.wikipedia.org/wiki/Patrimoine_culturel

${ }^{10}$ Le sixième projet de CREMI portant sur l'innovation patrimoniale.
} 
C'est aussi, saisir l'opportunité de cette évolution où le "plus être" doit relayer le "plus avoir" et créer à partir de sa culture, son sol, son histoire ce qui pourra devenir des ressources nouvelles, et en faire les vecteurs de son développement.

Les ressources patrimoniales ne sont pas, tout le temps, données, mais construites et le milieu joue dans cette construction. La connaissance est également importante en matière d'innovation patrimoniale. C'est elle qui donne au service toute sa valeur ajoutée. Etant ceci, une distinction soit à opérer en matière de ressources, au regard de la manière dont elles s'insèrent dans un système territorial avec attention particulière à porter aux caractéristiques territoriales des ressources.

\subsubsection{Deux classifications territoriales des ressources patrimoniales relevant des mêmes approches}

Dans la cohérence avec le sujet de cette recherche, les classifications classiques aux ressources patrimoniales, matérielles, immatérielles, culturelles, naturelles, architecturales ou autres, comptent moins par rapport aux critères territoriaux. Etant ceci, nous nous contentons de deux catégorisations, précisément celles reproduites dans les travaux de GREMI VI. La première, classant les ressources en "ressources génériques" et "ressources spécifiques" était proposée par Peyrache-Gadeau et Pecqueur $(2004)^{l 1}$, tandis que la deuxième était présentée dans le travail de Kebir et Crevoisier (2004) ${ }^{12}$, et fait distinction entre "ressources données" et "ressources construites".

La distinction de Peyrache-Gadeau et Pecqueur (2004) est guidée par deux caractères principaux, la "transférabilité" et la "spécificité" de la ressource :

- Ressources spécifiques : leur valeur dépend de leur incorporation dans un processus de production. Elles sont difficilement transférables, comme elles permettent au territoire de se différencier des autres, notamment par leur spécificité fortement "encastrée" dans leur territoire.

- Ressources génériques : contrairement aux ressources spécifiques, leur valeur n'est pas fonction de leur usage. Elles n'ont pas d'influence sur l'attractivité du territoire, ni sur sa compétitivité par différenciation, du fait qu'elles existent ailleurs ou que le coût de leur transfert est faiblement considérable.

Dans leur étude, Peyrache-Gadeau et Pecqueur (2004) ont également proposé une catégorisation, suivant l'usage de la ressource. Une "distinction simple entre ressources destinées à un ou à plusieurs types d'usages"13. Deux modes en découlent donc :

- Mode d'usage "simple".

- Mode d'usage "complexe".

Partant toujours des mêmes approches de classification, conceptualiser les ressources à partir d'une optique statique, en tant que stock et réserve ou dynamique en tant que construction et l'émanation d'un quelconque processus. Kebir et Crevoisier (2004) différencient entre "ressources données" et "ressources construites" :

\footnotetext{
${ }^{11}$ Véronique PEYRACHE-GADEAU et Bernard PECQUEUR, « Les ressources patrimoniales : une modalité de valorisation par les milieux innovateurs de ressources spécifiques latentes ou existantes ».

12 Leïla Kebir et Olivier Crevoisier, «Dynamique des ressources et milieux innovateurs ».

${ }^{13}$ Véronique PEYRACHE-GADEAU et Bernard PECQUEUR, « Les ressources patrimoniales : une modalité de valorisation par les milieux innovateurs de ressources spécifiques latentes ou existantes », 73.
} 
- Ressources données : elles existent indépendamment des systèmes de production. Souvent, elles sont transférables et homogènes.

- Ressources construites : à l'inverse des ressources données, elles sont évolutives et se construisent continuellement par le processus de production où elles sont inscrites.

Le milieu innovateur, par son rôle de régulateur évoqué ci-dessus (entre la logique marchande et le souci de durabilité), procède à l'inscription territoriale de la ressource de manières différentes. Le mode d'articulation est fonction des caractéristiques de la ressource et de son usage.

\subsubsection{Les configurations envisageables de l'innovation patrimoniale}

Différents processus d'inscription innovante des ressources patrimoniales dans un système territorial de production, peuvent être opérés :

- Valorisation d'une ressource spécifique, contextualisée ou dédiée, ou d'une ressource générique.

- Requalification d'une ressource.

- Mise en œuvre de la valorisation de la ressource à partir d'une ressource latente.

C'est en relation avec ses processus qu'il faut aborder le rôle du milieu. Le milieu peut aussi, avoir à faire face à une situation où prédominent des comportements individualistes et opportunistes qui pourraient le déstructurer et le faire disparaitre. Le milieu innovateur apparait en effet, comme le régulateur des diverses tensions qui résultent de la nécessité d'assurer à la fois, la pérennité de la ressource et le fonctionnement efficace et rentable des systèmes territoriaux de production.

L'articulation entre ressource et système de production débouche sur la classification suivante :

- Ressource standard ou générique.

- Ressource "donnée" ou issue de la dynamique territoriale.

C'est l'hypothèse de base pour élargir la notion de milieu innovant au-delà de l'industrie et des services et au-delà de la ville. Assurément, l'attractivité d'un territoire ainsi que sa durabilité sont d'autant plus fortes qu'il dispose de ressources et d'avantages spécifiques, non susceptibles d'exister ailleurs ou d'être dupliquées et échappent, conséquemment, à la concurrence par le marché.

Les ressources ne sont plus considérées pour elles-mêmes ni même pour leur capacité à s'intégrer dans une combinatoire donnée de facteurs, mais elles deviennent l'objet d'un processus de "requalification" qui engage un certain état de forme des coordinations, des expériences antérieures de coordination, et à une certaine "densité" institutionnelle au plan territorial.

Une nouvelle distinction est à faire, non par leur caractère plus au moins transférable et reproductible dans l'espace, mais aussi par leur mode de mise en valeur (destination), simple avec un seul type d'usage ou complexe en cas de pluralité d'usages. Etant ceci, quatre configurations sont envisageables : 


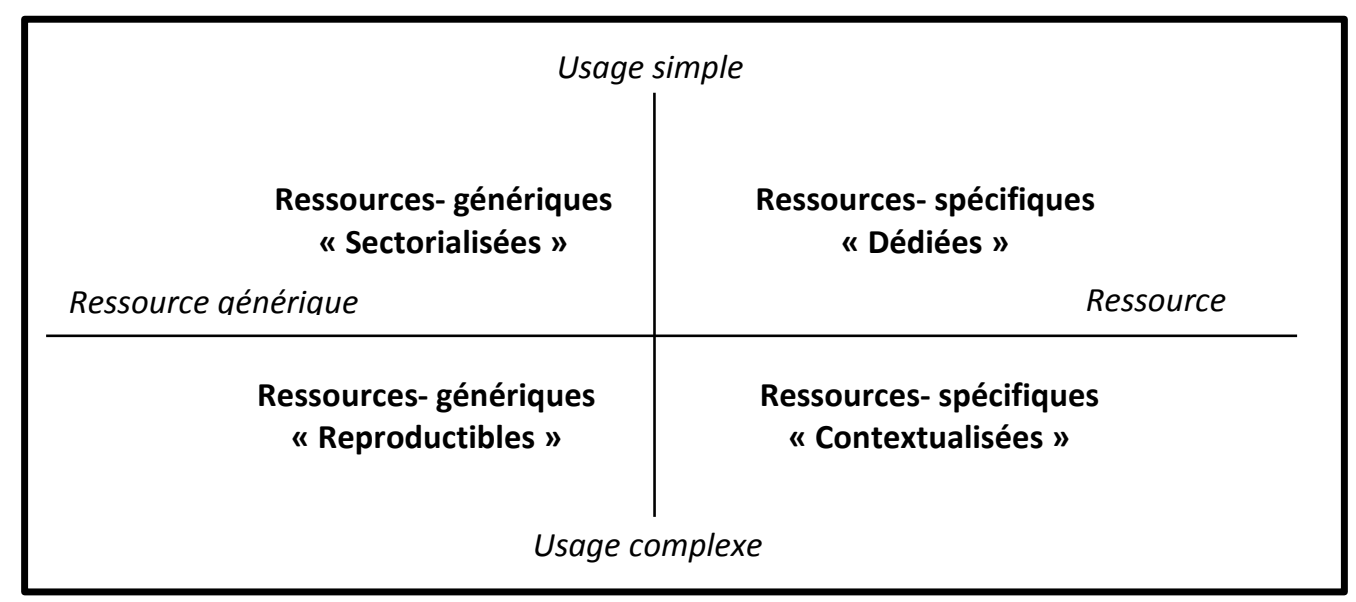

Figure 4 : Les configurations possibles des ressources

\subsection{Les dynamiques des ressources patrimoniales}

\subsubsection{Approche standard, ressources données comme étant stock fini et don exogène au processus de production.}

Comment allouer pertinemment et efficacement les ressources existantes compte tenu des visées du territoire?

La rareté est bien la pierre angulaire de la réflexion. Williamson (1985) (néo institutionnaliste) remet en question, le postulat d'homogénéité lorsqu'il observe l'existence d'un actif spécifique qui engage un coût de transaction en cas de réaffectation. Et donc d'autres formes d'allocation sont à considérer notamment la hiérarchie ou le réseau (intégration dans le cache d'une organisation) faisant d'elle une donnée de l'histoire.

Pour les néoclassiques, l'équilibre s'établit de manière instantanée et s'effectue dans un espace qui se réduit à un point, mais rien n'est dit sur l'enchainement des ressources et les processus de production et leur articulation dans les différentes échelles, spatiales et temporelles.

\subsubsection{Approche constructive, la ressource comme construite résultante d'un processus.}

Une ressource est une relation entre un acteur, une pratique et une matière (Raffestin, 1980). La pratique sur la ressource évolue dans le temps et dans l'espace et s'appuie sur des connaissances accumulées. La seconde conception des ressources et celle institutionnelle et évolutionniste s'interrogeant plus sur l'inscription territoriale de la ressource.

La ressource occupe et compose un espace-temps. Cet espace, résulte de l'interaction entre la ressource en question et le contexte dans lequel elle se développe. Les processus qui définissent une ressource se déroulent à partir d'un territoire qui constitue au départ, la matrice de ces relations. Réciproquement, ce dernier processus produit une empreinte sur le territoire qui devient à son tour la matrice du développement, et ainsi de suite ...

Les problématiques autour des ressources patrimoniales doivent être décomposées en trois dimensions ${ }^{14}$ :

${ }^{14}$ Olivier Crevoisier, "L'approche par les milieux innovateurs : état des lieux et perspectives ». 
- Dimension technologique : apprentissage et développement technologique au niveau des marchés, systèmes de production, identification des ressources, leur mise en valeur...

- Dimension organisationnelle : les interactions et le réseau d'acteurs. La manière dont les ressources sont allouées entre les acteurs. Les ressources peuvent être générées de manière durable non par le marché, mais par des règles de concurrence/coopération.

- Dimension institutionnelle : surtout les relations de proximité et le territoire. La reconversion des ressources dans un produit ou un service innovateur n'est pas possible sans accès à cette ressource. Dans quelle mesure la proximité influence-t-elle la création/destruction des ressources?

Dans le perspectif "milieu innovateur", la manière dont s'enchainent dans le temps et dans l'espace, les processus de création et de destruction, d'identification et d'actualisation des ressources dans et par le système de production, façonnent les dynamiques générées au niveau du territoire. L'activité des systèmes de production affecte les objets (ressources) par création/destruction, ce qui conditionne le développement des systèmes de production.

La ressource est bien l'ensemble des objets identifiés comme pouvant s'inscrire dans un processus de production de bien ou de service. Elle est conçue comme un ensemble de quatre processus :

- Création /destruction (l'objet).

- Identification et actualisation (manière dont les ressources sont incorporées dans le système de production).

L'objet et les systèmes de production agissent les uns sur les autres ce qui nécessite de mettre en place des modes et des formes organisationnelles de coordination (marché, réseau, milieux) et de gouvernance. Dans le temps et dans l'espace, les changements dans les systèmes de production vont de pair avec des couplages, découplage ou recouplage qui transforment les objets en ressources et réciproquement dans le sens inverse.

Dans l'approche standard, les ressources préexistent au processus de production. Or, nous observons que des ressources telles que les connaissances ne précédent pas forcement la production, ils peuvent naitre au moment de la production ou même postérieurement.

Certains objets, peuvent se déplacer dans l'espace, certaines sont forcément ancrés soit en amont soit en aval car leur spécificité et leur mobilité doivent se faire au sein d'un système de production particulier.

\subsubsection{Typologies des dynamiques territoriales des ressources}

La dynamique dépend de la façon dont les processus de création, destruction, identification et actualisation s'enchainent et s'effectuent mutuellement au sein d'un territoire concret et donc de la forme des interrelations objet/système de production.

Quatre dynamiques sont envisageables :

- Dynamique de croissances renouvelables : la dynamique des systèmes de production entraine positivement celle de l'objet.

- Dynamique d'érosion et/ou d'épuisement : la dynamique de systèmes de production affecte négativement celle de l'objet. La ressource régresse. 
- La mise en valeur : la dynamique de l'objet entraine positivement celle du système de production.

- La pénurie : la dynamique de l'objet affecte négativement celle des systèmes de production.

La pensée économique contemporaine, et également les "régiologistes" (Desroches, 2012) cherchent à comprendre et à expliquer le développement territorial, mettant en corrélation les inputs possibles, notamment l'innovation, le système d'acteurs et le mode de régulation d'un côté, et les changements économiques, sociales et écologiques de l'autre côté.

L'analyse par milieux innovateurs, spécialement par approche de l'innovation patrimoniale offre un nouveau cadre d'analyse et un nouveau regard sur les modèles de développement. Les processus qui s'enclenchent par exploitation des ressources patrimoniales ont un impact croisé et de dynamisation mutuelle, entre la ressource et son milieu. Plus particulièrement en cas de dynamique de croissance renouvelable ou de mise en valeur, le développement simultané, et de la ressource et de son système de production garantit une articulation meilleure entre le caractère marchand de l'exploitation et le souci de sauvegarde de la ressource. Pourtant, la dynamique territoriale peut être néfaste au niveau organisationnel, à cause des nouveaux conflits d'intérêt ou de compétences entre les différentes parties prenantes du système de production.

Ajoutant, une dynamique qui pousse à la surexploitation de la ressource/l'objet, menace sa durabilité et par conséquence l'équilibre du milieu producteur. Le milieu innovateur peut être victime de son succès, par une dégradation subie de ses ressources ou l'augmentation excessive de l'offre ou de la demande sur le territoire.

Ces processus destructeurs conduisent, en retour, à d'autres dynamiques handicapantes affectant la ressource ou le système de production. Devant cet état de figure, d'autres formes d'innovation patrimoniale sont nécessaires pour retrouver l'équilibre et redynamiser la machine productive locale.

\section{Conclusion}

L'apport espéré de ce papier réside en prime abord, dans les éclaircissements qui porte à la notion de l'innovation patrimoniale par des ressources culturelles et naturelles. Deuxièmement, l'analyse des relations causales qui naissent entre les innovations et la dynamique économique du territoire.

L'étude des processus de valorisation des ressources naturelles et/ou culturelles induit une conception élargie des modalités d'innovation. Particulièrement, la capacité à identifier des ressources latentes et la requalification des ressources existantes pour des fins de développement territorial durable. En outre, l'analyse par le "local" permet de saisir le milieu comme une variable qui conditionne la durabilité.

Les ressources sont des enchainements de processus complexes, évolutifs, dynamiques et parfois contradictoires. Leur inscription territoriale peut varier au fil de l'évolution des objets et des systèmes de production concernés.

La relation entre la dynamique des ressources et l'évolution des systèmes de production territoriaux soulève de manière plus fondamentale, la question des interactions entre l'économique, le reste de la société et la nature. Différentes configurations de mise en valeur de la ressource patrimoniale sont envisageables en fonction des caractéristiques des ressources et des modes de leur incorporation dans des systèmes de production. 
En plus, les pratiques et l'exploitation innovante donnent lieu à d'autres types de dynamiques des ressources patrimoniales, distinction faite entre l'objet de la ressource et le système de production auquel a été utilisée. Néanmoins, un approfondissement est requis quant aux menaces que peuvent produire les tensions entre les institutions du système de production, ou suite aux interactions entre la ressource et les systèmes sociotechniques et socio-écologiques du milieu innovateur.

\section{BIBLIOGRAPHIE}

[1] Andrée Matteaccioli, et Muriel Tabariés, "Historique du GREM”,GREMI-Université de Neuchâtel, 2006.

[2] Denis Maillat, et Jean-Claude Perrin., "Entreprises innovatrices et développement territorial ", Université de Neuchâtel Neuchâtel: GREMI/EDES, 1992.

[3] Denis MAILLAT, Michel Quévit, et Lanfranco Senn, "Réseaux d'innovation et milieux innovateurs : un pari pour le développement régional”, Neuchâtel: GREMI/EDES, 1993.

[4] Dimitri Uzunidis, "Milieu innovateur, relation de proximité et entrepreneuriat. Analyse d'une alchimie féconde", Revue canadienne des sciences régionales, no 33 (2010), pp.91-106.

[5] Jean-Claude Perrin, "Pour une révision de la science régionale. L'approche par les milieux", Revue canadienne des sciences régionales XV, no 2 (1992), pp. 155-97.

[6] Leïla Kebir, et Olivier Crevoisier, "Dynamique des ressources et milieux innovateurs", In Ressources naturelles et culturelles, milieux et développement local, 261-90. Neuchâtel: EDES-GREMI, 2004.

[7] Michel Quévit, "Milieux innovateurs et couplage local- international dans les stratégies d'entreprise: un cadre pour l'analyse", Revue canadienne des sciences régionales XV, no 2 (1992), pp.219-37.

[8] Muriel TABARIES, “Les apports du GREMI à l'analyse territoriale de l'innovation ou 20 ans de recherche sur les milieux innovateurs", Matisse-Cahiers de la MSH, 2005, Centre National de la Recherche Scientifique édition.

[9] Olivier Crevoisier, "L’approche par les milieux innovateurs : état des lieux et perspectives ». Revue d’Économie Régionale \& Urbaine 2001/1(février) (2001), pp.153-65.

[10] Véronique PEYRACHE-GADEAU, et Bernard PECQUEUR, "Les ressources patrimoniales: une modalité de valorisation par les milieux innovateurs de ressources spécifiques latentes ou existantes", In Ressources naturelles et culturelles, milieux et développement local, 71-90. Neuchâtel: EDES-GREMI, 2004. 